\title{
Quantitative Analysis of Collagens and Fibronectin Expression in Human Right Ventricular Hypertrophy ${ }^{a}$
}

\author{
T.H.F. PETERS, ${ }^{b, c}$ H.S. SHARMA, ${ }^{b, d}$ E. YILMAZ, ${ }^{b}$ AND A.J.J.C. BOGERS ${ }^{c}$ \\ ${ }^{b}$ Pharmacology, ${ }^{c}$ Cardiothoracic Surgery, Erasmus University Medical Center, \\ Rotterdam, The Netherlands
}

\begin{abstract}
One of the main features in human tetralogy of Fallot (TF) is right ventricular hypertrophy $(\mathrm{RVH})$ due to pressure (sub-pulmonary stenosis) and volume overload (ventricular septal defect). Currently, primary correction at a young age is the treatment of choice. To unravel the role of extracellular matrix in RVH, we examined myocardial expression of collagens and fibronectin in TF patients with primary correction (TF1, age $0.7 \pm 0.2 \mathrm{yr}$.), secondary surgery (TF2, age $36.9 \pm 4.6 \mathrm{yr}$ ), and in age-matched control patients. Sirius red staining quantified by video imaging showed significantly increased interstitial staining for collagens in both TF1 and TF2 groups as compared to respective controls. Fibronectin was expressed in extracellular spaces, perivascular regions, and in some cardiomyocytes. Quantitative analysis of fibronectin revealed increased expression in only TF1 group as compared to respective control. Our results indicate an increased amount of myocardial extracellular matrix deposition as a sign of fibrosis during RVH in patients with TF.
\end{abstract}

\section{INTRODUCTION}

Tetralogy of Fallot (TF) is a common form of cyanotic congenital heart disease, with an incidence rate of 1 out of 2,000 newborns. TF is characterized by overriding of the aorta, a ventricular septal defect, (sub)valvular pulmonary stenosis, and right ventricular hypertrophy $(\mathrm{RVH})$, resulting in hypoxemia due to diminished pulmonary flow. ${ }^{1}$ The RVH in human TF is an adaptive response of cardiomyocytes to increased pressure as well as volume overload caused by (sub)pulmonary stenosis and by ventricular septal defect, respectively. The adaptive response requires proportionate increase of coronary flow or growth of the coronary vasculature, leading to myocardial remodeling as well as development of fibrosis and, without treatment, ultimately results in cardiac failure. ${ }^{2-5}$

In myocardial fibrosis, collagens and fibronectin play a prominent role. Damage of the collagen structure compromises the myocyte support, decreases myocardial strength and stiffness, and allows expansion of the tissue. ${ }^{6}$ Collagen fibers are organized in the collagen network, which is found in the extracellular space of the myo-

${ }^{a}$ This work is supported by the Netherlands Heart Foundation (NHS 96.082).

${ }^{d}$ Address correspondence to: Hari S. Sharma, Ph.D., Institute of Pharmacology, Erasmus University, P.O. Box 1738, 3000 DR Rotterdam, The Netherlands; Telephone: 3110 4087963; Fax: 3110 4089458; E-mail: SHARMA@FARMA.FGG.EUR.NL 
cardium. ${ }^{7-10}$ In myocardial fibrosis a disproportionate accumulation, either reactive or reparative, of collagen has been observed. Experimental ${ }^{11}$ and clinical data ${ }^{12,13}$ show that a rise in collagen content increases myocardial stiffness and promotes abnormalities of cardiac function during hypertrophy. In a morphometrical study, an increase in fibrosis in TF was associated with an increased diameter of myocytes and an increase of myocardial disarray. ${ }^{14}$ In addition, the perivascular accumulation of collagen fibers may impair the vasodilator capacity of intramyocardial coronary arteries and may contribute to the decrease in coronary reserve. ${ }^{15}$

Fibronectin is found in the extracellular matrix of most tissue, serving as a bridge between cells and interstitial collagen mesh network and influencing diverse processes, including cell growth, adhesion, migration, and wound repair. ${ }^{16}$ In rats, fetal fibronectin was shown to have accumulated during development of cardiac hypertrophy by pressure overload. ${ }^{17}$ The hypertrophied heart exhibits significant qualitative as well as quantitative changes in gene expression. The altered expression patterns in hypertrophy include transient expression of proto-oncogenes. ${ }^{18}$ It is thought that those changes in cardiac hypertrophy reflect a change toward an embryonic program of gene expression. ${ }^{19}$ However, it is still not clear whether those altered expression patterns are general markers of cardiac hypertrophy or markers of a specific parallel pathogenic process, such as TF. Animal studies have shown that the levels of mRNA for fibronectin and collagens were elevated in cardiac hypertrophy. ${ }^{17}$

Most attention in research on human TF has so far focused on clinical treatment and surgical methods to limit the myocardial injury. TF patients are usually operated on during their first year of life. The aim of early primary repair is to close the ventricular septal defect and to remove the right ventricular outflow tract obstruction, thus relieving the hypoxemia and eliminating the stimulus for the adaptive $\mathrm{RVH}$ as well as preserving right ventricular function. ${ }^{1,20}$ However, after corrective surgery some patients develop right ventricular failure due to volume-overload caused by pulmonary regurgitation and may for this reason require further secondary corrective surgery later in life. ${ }^{21,22}$ Histopathological studies on TF myocardial tissue reported that the myocardial cell diameter was reduced in patients after corrective surgery, indicating regression of RVH. Nevertheless, it is not yet known to what extent the RVH in TF patients regresses. Little attention has been paid to the molecular component of this disease. In this study, we examined the degree of fibrosis in human TF by measuring the expression of myocardial fibrosis markers, such as fibronectin and collagen, in right ventricular biopsies obtained during corrective surgery for $\mathrm{TF}$ and comparing the data with that of age-matched patients with normal right ventricle.

\section{MATERIALS AND METHODS}

\section{Biopsies}

The present study was approved by the Medical Ethical Committee of the University Hospital, Erasmus University, Rotterdam. Myocardial tissue biopsies were obtained from patients who underwent primary corrective surgery (TF1, mean age $0.7 \pm 0.2 \mathrm{yr}, N=11$ ), secondary surgery (TF2, mean age $36.9 \pm 4.6 \mathrm{yr}, N=6$ ), and age-matched control patients. All control patients had clinically normal right ventricle and underwent autograft aortic root replacement $(\mathrm{C} 1$, mean age $1.5 \pm 0.2 \mathrm{yr}, N=$ 
6 and $\mathrm{C} 2$, mean age $31.7 \pm 4.2 \mathrm{yr}, N=12$ ). Myocardial tissue was fixed in $4 \%$ paraformaldehyde in PBS for minimal $24 \mathrm{~h}$ and further processed for dehydration and embedding in paraffin for histological and immunohistochemical studies. ${ }^{23}$

\section{Sirius Red Staining}

The total collagen in myocardial tissue specimens was stained with Sirius red F3BA. ${ }^{24}$ Tissue sections of $6 \mu \mathrm{m}$ thickness, were treated with $0.2 \%$ aqueous phosphomolybdic acid and incubated in $0.1 \%$ Sirius red. Before dehydration, the slides were treated with $0.01 \mathrm{~N} \mathrm{HCl}$ and mounted. The Sirius red-stained area was determined as percentage of total tissue area using a Kontron-KS 400 (Kontron Electronik/Zeiss, Eching, Germany) computerized image analysis system. Distribution of collagen fibers in the interstitial space as well as in the perivascular region was measured as Sirius red-positive area. Twelve different images from each section were analyzed to calculate the mean percentage of stained tissue area and statistically significant values were accepted at $p \leq 0.05$.

\section{Immunohistochemistry}

After fixation and dehydration, the cardiac tissue was embedded in paraffin. Sections of $6 \mu \mathrm{m}$ thickness were cut and mounted on poly-L-lysine-coated microscope slides, followed by immunohistochemistry using the avidin-biotin complex (ABC) method (BioGenex, San Ramon, CA). The sections were deparaffinized and quenched for endogenous peroxidase by $2 \%$ hydrogen peroxide in methanol. Nonspecific binding sites were blocked by $10 \%$ normal goat serum. Human serum preabsorbed polyclonal antibodies, against human fibronectin in 1:1,000 dilution (Life Technologies, Breda, The Netherlands), were applied as primary antiserum and the sections were incubated at room temperature for $30 \mathrm{~min}$. Negative controls were performed by omission of the primary antibody. After washing in $0.5 \%$ Tween-20 in PBS solution, the sections were incubated with mouse biotinylated anti-rabbit IgG (BioGenex, San Ramon, CA) for 30 min and subsequently, with peroxidase conjugated streptavidin. Color was developed using 3,3'-diaminobenzidine tetrahydrochloride dehydrate (DAB) and then sections were counter-stained with hematoxylin. Sections were mounted and visualized under the light microscope and photographed. A semiquantitative analysis of the sections was done by two independent observers using a staining score ranging from 0 (no staining) to 4 (very strong, dark brown, staining) to assess the level of fibronectin expression in the myocardial tissue obtained from TF patients and age-matched controls.

FIGURE 1. Right ventricular total collagen content in patients with tetralogy of Fallot. Micrographs of right ventricular tissue obtained from patients undergoing surgery (A) for autograft aortic root replacement (Control), (B) for primary repair due to tetralogy of Fallot (TF1), and (C) for secondary surgery (TF2). Tissue sections were prepared and stained with collagen-specific stain, Sirius red. Collagen fibers appear red and are localized in interstitial and perivascular areas. Note the interstitial fibrosis in the case of TF patients. Bar $=250 \mu \mathrm{m}$. (D) Significantly enhanced interstitial collagen content in TF1 group as compared to the control. Distribution of collagen fibers in tissue was visualized under normal and polarized light and quantified using video imaging software. Values are fold induction and shown as mean \pm SEM of sirius red-positive interstitial/total tissue area (arrows). 




\section{RESULTS AND DISCUSSION}

Sirius red staining was performed for the total collagens in order to assess the degree of myocardial fibrosis. The red staining was quantified by video imaging and showed a significantly increased interstitial and perivascular staining for total collagens in TF1 (1.4 \pm 0.2 fold) (FIG. 1A and D) and TF2 (1.6 \pm 0.2 fold) (FIG. 1B and 1D) as compared to their respective controls (FIG. 1C and 1D). The immunolocalization of fibronectin showed that fibronectin was expressed in the interstitium, in the perivascular area, as well as in some cardiomyocytes. Semiquantitative analysis using a staining score ranging from $0-4$, revealed that the fibronectin expression was significantly increased in TF1 (FIG. 2A and 2D) as compared to control (3.0 \pm 0.2 versus $2.6 \pm 0.2$ ). There were no differences in fibronectin expression in patients from TF2 (FIG. 2B and 2D) as compared with age-matched controls (FIG. 2C and 2D). Our results indicate an increased amount of myocardial extracellular matrix deposition in RVH in patients with TF, with an apparent increase of total collagen and a slight increase in fibronectin at young age.

The apparent development of cardiac hypertrophy is induced by changes in cardiac gene expression that provide the heart a means of compensation for increased hemodynamic load. ${ }^{25}$ In this regard, our data are in agreement with the histopathologic findings from the RV myocardium in preoperative TF, which show interstitial fibrosis, myofibrillar disorganization, disarray, and degenerative changes, in addition to myocardial cell hypertrophy. ${ }^{18,26,27}$ The changes found in our study of expression of collagens and fibronectin could be attributed to altered structure or function of the myocardium. It is believed that changes in gene expression accompanying cardiac hypertrophy are the result of a multifactorial process and the observed changes may therefore be concomitant phenomena. The alteration in ventricular gene expression might be an indicator of cardiac hypertrophy and may result from a number of different stimuli. We assume that the changes in the right ventricular fibronectin and collagen expression in TF patients could be as a result of increased hemodynamic load as it has been often found to be parallel with the increase in ventricular mass. ${ }^{19}$

In the case of TF patients, the early increase of collagen and fibronectin deposition in the right ventricle may occur because the original myocardial extracellular matrix has been expanded and weakened due to the hemodynamic overload. The myocardium will compensate until enough new collagen has been produced to restore tensile strength and to resist the distending forces. Our findings in the TF1 and TF2 groups fit into this concept as the TF2 shows a more prominent increase in the

FIGURE 2. Immunohistochemical localization of fibronectin in right ventricular tissue of patients with tetralogy of Fallot. Micrographs showing human right ventricular tissue stained with rabbit-anti-human fibronectin polyclonal antibodies (as described in Materials and Methods) from patients undergoing surgery (A) for autograft aortic root replacement (Control), (B) for primary repair for tetralogy of Fallot (TF1), and (C) for secondary surgery (TF2). Bar $=500 \mu \mathrm{m}$. (D) Significantly enhanced interstitial fibronectin staining in the TF1 group as compared to the respective controls. A semiquantitative analysis of the section was done by two independent observers using a staining score ranging from 0 (no staining) to 4 (very strong, dark brown, staining) to assess the levels of fibronectin staining (arrows). Values are shown as mean \pm SEM. 


amount of collagen as compared to TF1. Corrective surgery limits the myocardial overload and may therefore limit the amount of damage to the myocardial collagen matrix. ${ }^{6}$ Our study provides further evidence that myocardial architecture in patients with $\mathrm{TF}$ is altered towards fibrotic state as the expression levels of extracellular matrix components like collagens and fibronectin were elevated. However, further research at the mRNA level would add to the notion that the gene expression of a number of proteins involved in fibrosis was transcriptionally altered.

\section{SUMMARY AND CONCLUSION}

In the present study the degree of myocardial fibrosis at protein level in patients with tetralogy of Fallot (TF) was examined by assessing quantitatively the expression of total collagens and fibronectin. We found a significant increase in staining for interstitial and total collagens at primary and secondary corrective surgery as compared to the age-matched control patients. However, immunohistochemical localization of fibronectin revealed significant increase in this protein levels at primary correction only. Our results clearly demonstrate an increased amount of myocardial extracellular matrix deposition resulting in tissue fibrosis in TF patients with right ventricular hypertrophy. This vital information could be of great help in assessing the timing of surgery as well as post-operative prognosis.

\section{REFERENCES}

1. Castaneda, A.R. et al. 1994. Cardiac Surgery of Neonate and Infant: 215-234. Saunders. Philadelphia.

2. ARAI, M. et al. 1994. Sarcoplasmic reticulum gene expression in cardiac hypertrophy and heart failure. Circ. Res. 74: 555-564.

3. VAN BILSEN, M. \& K.R. ChIEN. 1993. Growth and hypertrophy of the heart: towards an understanding of cardiac specific and inducible gene expression. Cardiovasc. Res. 27: $1140-1149$.

4. Boluyt, M.O. et al. 1994. Alterations in cardiac gene expression during the transition from stable hypertrophy to heart failure. Marked upregulation of genes encoding extracellular matrix components. Circ. Res. 75: 23-32.

5. SCHwARTZ, K. et al. 1993. Molecular phenotype of hypertrophied and failing myocardium. Circulation 87: VII-5-VII-10.

6. WhitTAKeR, P. 1997. Collagen and ventricular remodeling after acute myocardial infarcion: concepts and hypotheses. Basic Res. Cardiol. 92: 79-81.

7. Weber, K.T. et al. 1994. Collagen network of the myocardium: function, structural remodeling and regulatory mechanisms. J. Mol. Cell Cardiol. 26: 279-292.

8. BISHOP, J.E. et al. 1994. Increased collagen synthesis and decreased collagen degradation in right ventricular hypertrophy induced by pressure overload. Cardiovasc. Res. 28: $1581-1585$.

9. BRILla, C.G. et al. 1991. Impaired diastolic function and coronary reserve in genetic hypertension. Role of interstitial fibrosis and medial thickening of intramyocardial coronary arteries. Circ. Res. 69: 107-115.

10. Chapman, D. et al. 1990. Regulation of fibrillar collagen types I and III and basement membrane type IV collagen gene expression in pressure overloaded rat myocardium. Circ. Res. 67: 787-794.

11. JALIL, J.E. et al. 1989. Fibrillar collagen and myocardial stiffness in the intact hypertrophied rat left ventricle. Circ. Res. 64: 1041-1050. 
12. Hess, O.M. et al. 1981. Diastolic function and myocardial structure in patients with myocardial hypertrophy. Special reference to normalized viscoelastic data. Circulation 63: $360-371$.

13. Mclenachan, J.M. \& H.J. Dargie. 1990. Ventricular arrhythmias in hypertensive left ventricular hypertrophy. Relationship to coronary artery disease, left ventricular dysfunction, and myocardial fibrosis. Am. J. Hypertens. 3: 735-740.

14. KawAI, S. et al. 1984. A morphometrical study of myocardial disarray associated with right ventricular outflow tract obstruction. Jpn. Circ. J. 48: 445-456.

15. Strauer, B.E. 1990. The significance of coronary reserve in clinical heart disease. J. Am. Coll. Cardiol. 15: 775-783.

16. Farhadian, F. et al. 1995. Fibronectin expression during physiological and pathological cardiac growth. J. Mol. Cell. Cardiol. 27: 981-990.

17. Samuel, J.L. et al. 1991. Accumulation of fetal fibronectin mRNAs during the development of rat cardiac hypertrophy induced by pressure overload. J. Clin. Invest. 88: $1737-1746$.

18. BRAnd, T. et al. 1992. Proto-oncogene expression in porcine myocardium subjected to ischemia and reperfusion. Circ. Res. 71: 1351-1360.

19. Vikstrom, K.L. et al. 1998. Hypertrophy, pathology, and molecular markers of cardiac pathogenesis. Circ. Res. 82: 773-778.

20. Starnes, V.A. et al. 1994. Current surgical management of tetralogy of Fallot. Ann. Thorac. Surg. 58: 211-215.

21. Murphy, J.G. et al. 1993. Long-term outcome in patients undergoing surgical repair of tetralogy of Fallot. N. Engl. J. Med. 329: 593-599.

22. WARNER, K.G. et al. 1993. Restoration of the pulmonary valve reduces right ventricular volume overload after previous repair of tetralogy of Fallot. Circulation 88: II189-197.

23. Sharma, H.S. et al. 1994. Expression and immunohistochemical localization of vascular endothelial growth factor during ischemia induced ventricular dysfunction in pigs. Circulation 90: I-522.

24. TAKahashi, T. et al. 1992. Age-related differences in the expression of proto-oncogene and contractile protein genes in response to pressure overload in the rat myocardium. J. Clin. Invest. 89: 939-946.

25. KAtz, A.M. 1990. Cardiomyopathy of overload. A major determinant of prognosis in congestive heart failure. N. Engl. J. Med. 322: 100-110.

26. Mitsuno, M. et al. 1993. Fate of right ventricular hypertrophy in tetralogy of Fallot after corrective surgery. Am. J. Cardiol. 72: 694-698.

27. BRAND, T. et al. 1993. Expression of nuclear proto-oncogenes in isoproterenolinduced cardiac hypertrophy. J. Mol. Cell. Cardiol. 25: 1325-1337. 